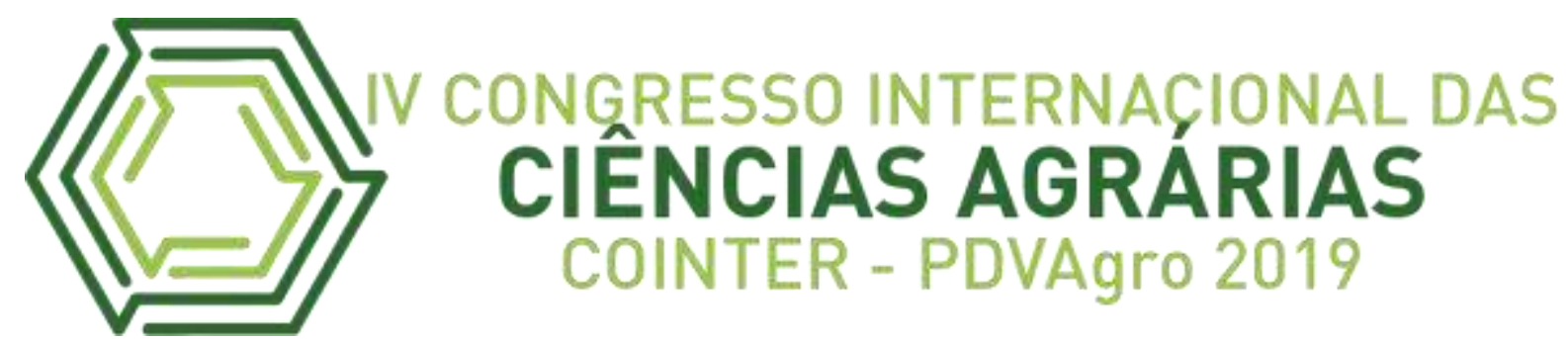

\title{
ANÁLISE DA PRODUÇÃO E DO COEFICIENTE DE DECOMPOSIÇÃO DE LITEIRA EM TRÊS DIFERENTES SISTEMAS DE MANEJO
}

\section{ANALYSIS OF THE PRODUCTION AND COEFFICIENT OF LITTER DECOMPOSITION IN THREE DIFFERENT MANAGEMENT SYSTEMS}

\author{
Apresentação: Comunicação Oral
}

Joélen Carla Pastana da Silva ${ }^{1}$; Antonia Cristiane Lobo Santana ${ }^{2}$; Jandson José do Vale Guimarães $^{3}$; Denise de Andrade Cunha ${ }^{4}$; Roberta de Fátima Rodrigues Coelho ${ }^{5}$ DOI :https://doi.org/10.31692/2526-7701.IVCOINTERPDVAgro.2019.0153

\section{Resumo}

A liteira é uma camada superficial composta por materiais de partes dos vegetais, como: folhas, fragmentos de caules, estruturas reprodutivas e materiais não identificados. A velocidade de decomposição da liteira regula o acúmulo de matéria orgânica na superfície do solo e o processo de ciclagem de nutrientes. $\mathrm{O}$ objetivo do presente trabalho foi analisar quantitativamente a produção de liteira e o seu coeficiente de decomposição em três diferentes sistemas de manejo (um Sistema Agroflorestal, um sistema de monocultivo de Citros e um fragmento florestal) localizados no Instituto Federal de Educação, Ciência e Tecnologia (IFPA) Campus Castanhal. A coleta de dados foi realizada em 2 parcelas de $30 \mathrm{~m}$ x 30m, em cada sistema de manejo. Para a coleta da produção de liteira foram instalados 4 coletores de $1 \mathrm{~m}^{2}$, em cada sistema, monitorados em intervalos de 20 dias, durante 60 dias. O coeficiente de decomposição foi considerado como a razão entre a liteira produzida e a liteira acumulada (caracterizada como aquela que fica depositada sobre o solo), a qual foi coletada em uma área delimitada de 0,50 m x 0,50 m em cada parcela. A liteira foi separada manualmente em duas frações: material lenhoso e não lenhoso; colocado para secar em estufa e, posteriormente, pesado. Houve maior produção

\footnotetext{
${ }^{1}$ Graduanda em Agronomia, Instituto Federal de Educação Ciência e Tecnologia do Pará - Campus Castanhal, joelemc6@gmail.com

${ }^{2}$ Graduanda em Agronomia, Instituto Federal de Educação Ciência e Tecnologia do Pará - Campus Castanhal, cristianelobosantana@gmail.com

${ }^{3}$ Graduando em Agronomia, Instituto Federal de Educação Ciência e Tecnologia do Pará - Campus Castanhal, guimaraesjandson@gmail.com

${ }^{4}$ Professora Dra. Bióloga, Instituto Federal de Educação Ciência e Tecnologia do Pará - Campus Castanhal, denise.cunha@ifpa.edu.br

${ }^{5}$ Professora Dra. Engenheira Florestal, Instituto Federal de Educação Ciência e Tecnologia do Pará - Campus Castanhal, roberta.fatimacoelho@gmail.com
} 
de liteira lenhosa no SAF. A Floresta apresentou maior produção de material não lenhoso acumulado. O coeficiente de decomposição do material não lenhoso acumulado manteve-se semelhante para todos os sistemas de manejo. Esses resultados corroboram para o entendimento de algumas questões dos processos ecológicos, como ciclagens de nutrientes, atrelada à nutrição vegetal, estimativa da intensidade do dinamismo microbiano do solo, além de correlacionar com os manejos culturais, quando necessários.

Palavras-Chave: Liteira, Ciclagem de nutrientes, SAF, Citros, Floresta.

\begin{abstract}
The litter is a surface layer composed of materials from plant parts such as leaves, stem fragments, reproductive structures and unidentified materials. The litter decomposition rate regulates the accumulation of organic matter on the soil surface and the nutrient cycling process. The objective of the present work was to quantitatively analyze litter production and its decomposition coefficient in three different management systems (an Agroforestry System, a Citrus monoculture system and a forest fragment) located at the Federal Institute of Education, Science and Technology. (IFPA) Castanhal Campus. Data collection was performed in 2 parcels $30 \mathrm{~m} \times 30 \mathrm{~m}$ in each management system. To collect litter production, 4 collectors of $1 \mathrm{~m}^{2}$ were installed in each system, monitored at intervals of 20 days for 60 days. The decomposition coefficient was considered as the ratio between the produced litter and the accumulated litter (characterized as the one that is deposited on the ground), which was collected in a delimited area of $0.50 \mathrm{~m} \times 0.50 \mathrm{~m}$ in each plot. The litter was manually separated into two fractions: woody and not woody material; placed for oven drying and then weighed. There was a higher production of woody litter in the SAF. The forest presented higher production of accumulated not woody material. The decomposition coefficient of accumulated not woody material remained similar for all management systems. These results corroborate the understanding of some issues of ecological processes, such as nutrient cycling, linked to plant nutrition, estimation of the intensity of soil microbial dynamism, and correlate with crop management when necessary.
\end{abstract}

Keywords: Litter, Nutrient cycling, SAF, Citrus, Forest.

\title{
Introdução
}

A liteira, também conhecida como serapilheira, é uma camada superficial composta por parte de materiais dos vegetais, como: folhas, fragmentos de caules, estruturas reprodutivas e materiais não identificados. Essa matéria está intrinsecamente relacionada com a ciclagem de nutrientes, a qual ocorre no piso florestal e nas camadas mais internas do solo, proporcionando micro e macronutrientes, a partir da decomposição, para os seres que vivem no entorno e necessitam desses resíduos. (COSTA et al., 2010)

A decomposição de liteira, a qual se acumula sobre o solo nos ecossistemas florestais, é a principal via de transferência de nutrientes da vegetação para o solo. Assim, a qualidade da biomassa produzida e a taxa de decomposição das folhas de uma árvore podem ser 
determinantes para a fertilidade do solo e, consequentemente, para a produtividade de plantas abaixo de sua copa (PEIXOTO, 2017).

A disponibilidade e o padrão de ciclagem dos nutrientes constituem-se em um dos principais fatores limitantes para a produção primária e, evidentemente, para o estabelecimento e desenvolvimento dos ecossistemas. As estimativas dos estoques de nutrientes presentes nos compartimentos dos ecossistemas e as transferências existentes entre eles podem refletir as estratégias das comunidades frente aos tensores ambientais (DELLITI, 1995).

Dessa forma, o objetivo deste trabalho foi analisar a produção e o coeficiente de decomposição de liteira em três diferentes sistemas no Instituto Federal do Pará - campus Castanhal.

\section{Fundamentação Teórica}

A liteira é particularmente importante por atuar na superfície do solo como um sistema de entrada e saída, recebendo entradas via vegetação e, por sua vez, decompondo-se e suprindo o solo e as raízes com nutrientes e matéria orgânica, sendo essencial na restauração da fertilidade do solo em áreas em início de sucessão ecológica (EWEL, 1976). A decomposição dos resíduos orgânicos que formam a liteira é o principal processo de ciclagem de nutrientes em um ecossistema florestal (MONTAGNINI; JORDAN, 2002).

O processo em questão está grandemente relacionado com a manutenção do estoque de nutrientes minerais no solo. Os ecossistemas sobrevivem da ciclagem de nutrientes, a qual é determinada pela quantidade de minerais que entra, pela quantidade retida na vegetação, pelas taxas de decomposição dos diferentes componentes da matéria orgânica do solo e da liteira cuja inserção é pertinente nessa composição, pelas taxas de imobilização e mineralização de nutrientes e pela absorção dos nutrientes (CUEVAS; MEDINA, 1996). Qualquer intervenção na dinâmica desse processo pode modificar a sincronia entre a disponibilidade de nutrientes provenientes da decomposição dos resíduos vegetais e a demanda nutricional das plantas, gerando, na maioria das vezes, perdas de nutrientes do solo (MYERS et al., 1994). A ciclagem de nutrientes é um processo onde os elementos químicos permanecem no ecossistema e continuamente circulam entre os organismos e o ambiente físico (RICKLEFS, 2003).

A ciclagem de nutrientes tem uma extrema dependência por fatores externos, como as chuvas, que pode exercer um papel negativo quando em grandes intensidades, uma vez que estas lixiviam a camada de liteira e o solo, promovendo a remoção de nutrientes. Contudo, a 
água da chuva também exerce papel positivo ao promover a lavagem da copa das árvores e, dessa forma, levar ao solo o material vegetal. Ainda mais, promove a solubilização de nutrientes da liteira para o solo. (GAMA-RODRIGUES; MIRANDA, 1991).

Para facilitar esse processo, o ecossistema desenvolveu mecanismos adaptativos que permitem a retenção de nutrientes, destacando-se entre eles o desenvolvimento de uma camada de húmus (JORDAN; HERRERA, 1981; LAVELLE, 1987) e uma de raízes (JORDAN; HERRERA, 1981), que evita perdas por lixiviação (JORDAN; STARK, 1978). A liteira representa uma fonte de energia e de nutrientes para toda uma comunidade de organismos que realiza sua decomposição, liberando os nutrientes minerais e transformando em húmus as substâncias orgânicas mais refratárias à decomposição (ANDERSON; SWIFT, 1983).

A superfície do solo nos sistemas florestais nativos, encontra-se, geralmente, coberta por detritos orgânicos, principalmente de origem vegetal, coletivamente chamados de liteira (MARINO et. al., 1980). Ao cair no solo, os resíduos vegetais são logo decompostos, principalmente pela ação dos micros e macrorganismos do solo (LUIZÃO; SCHUBART, 1986; TEIXEIRA; BASTOS, 1989).

São chamados de processos fundamentais a produção e decomposição da liteira, e mais comumente mensurados, do fluxo de matéria orgânica e nutrientes da vegetação para a superfície do solo, sendo vitais para o funcionamento do ecossistema, principalmente, nas florestas tropicais situadas em solos pobres em nutrientes (GOLLEY, 1978).

A quantidade de liteira depositada é controlada por fatores como a latitude (BRAY; GORHAM, 1964), altitude, precipitação (LONDSDALE, 1988) e evapotranspiração (MEENTEMEYER et al. 1982), além dos aspectos edáficos (VITOUSEK, 1984), e biológicos, como a estrutura (SCHLITTLER et al., 1993; WERNECK et al., 2001), idade (LEITÃO FILHO et al. 1993) e composição florística da vegetação (SUNDARAPANDIAN; SWAMY, 1999). Além do mais, também podem afetar a produtividade do sistema. A intensidade com que cada fator atua está relacionada às características particulares da fitocenose (DELITTI, 1995).

Em condição de equilíbrio do ecossistema a taxa de decomposição pode ser estimada através da razão entre a quantidade de serapilheira produzida e a acumulada, não apresentando, esta última, variações significativas em sua quantidade absoluta (OLSON, 1963). A camada de liteira sobre o solo depende, além da produção, da velocidade de decomposição da matéria orgânica, que varia conforme a composição do substrato, atividade dos decompositores e das condições ambientais, particularmente, temperatura, umidade e propriedades físicas do solo 
(SPAIN, 1984).

A velocidade de decomposição da liteira regula o acúmulo de matéria orgânica (MO) na superfície do solo (HAAG, 1987; PEGADO et al., 2008) e o processo de ciclagem de nutrientes (ADAMS; ATTIWIL, 1986; COSTA et al, 2005; TEIXEIRA et al., 2012). Vários fatores controlam o mecanismo de decomposição, destacando-se como os de maior representatividade: a atividade da fauna do solo (ANDERSON et al., 1983; CÉSAR, 1993), as condições do ambiente (temperatura, precipitação pluviométrica e características edáficas do local), além da composição química dos resíduos (ABER; MELILO, 1978; TIAN et al., 1992; LANDSBERG; GOWER, 1997).

Para estimativa da taxa de decomposição da serapilheira utilizou-se a equação proposta por Olson (1963), empregada em estudos semelhantes (PAGANO, 1989; MORELLATO, 1992; CÉSAR, 1993): $\mathrm{K}=\mathrm{L} / \mathrm{X}$, em que $\mathrm{K}$ = coeficiente de decomposição, $\mathrm{L}$ = produção anual de serapilheira e $\mathrm{X}=$ média anual da serapilheira acumulada.

\section{Metodologia}

\section{Descrição da área de estudo}

O estudo foi desenvolvido em três diferentes sistemas de manejo localizados no Instituto Federal de Educação, Ciência e Tecnologia do Pará, localizado no município de Castanhal, Pará, no período de agosto a novembro de 2018 (Mapa 1). A pesquisa foi classificada de natureza quantitativa e do tipo experimental.

O município de Castanhal tem um clima tropical e uma pluviosidade significativa ao longo do ano. Mesmo o mês mais seco ainda assim tem muita pluviosidade. A classificação do clima é Af e a temperatura média é de $26.5^{\circ} \mathrm{C}$ de acordo com a Köppen e Geiger. Pluviosidade média anual de $2432 \mathrm{~mm}$. O mês mais seco é novembro com $63 \mathrm{~mm}$ e o mês mais quente do ano é outubro com temperatura média de $27.0^{\circ} \mathrm{C}$. (CLIMATE, 2018). O solo foi classificado em Latossolo Amarelo Distrófico típico, textura média (FERREIRA, 2008). 
Mapa 1. Mapa de localização: a) Floresta b) SAF c) Citros d) Município de Castanhal em relação aos coletores e) IFPA Castanhal em relacão aos coletores.

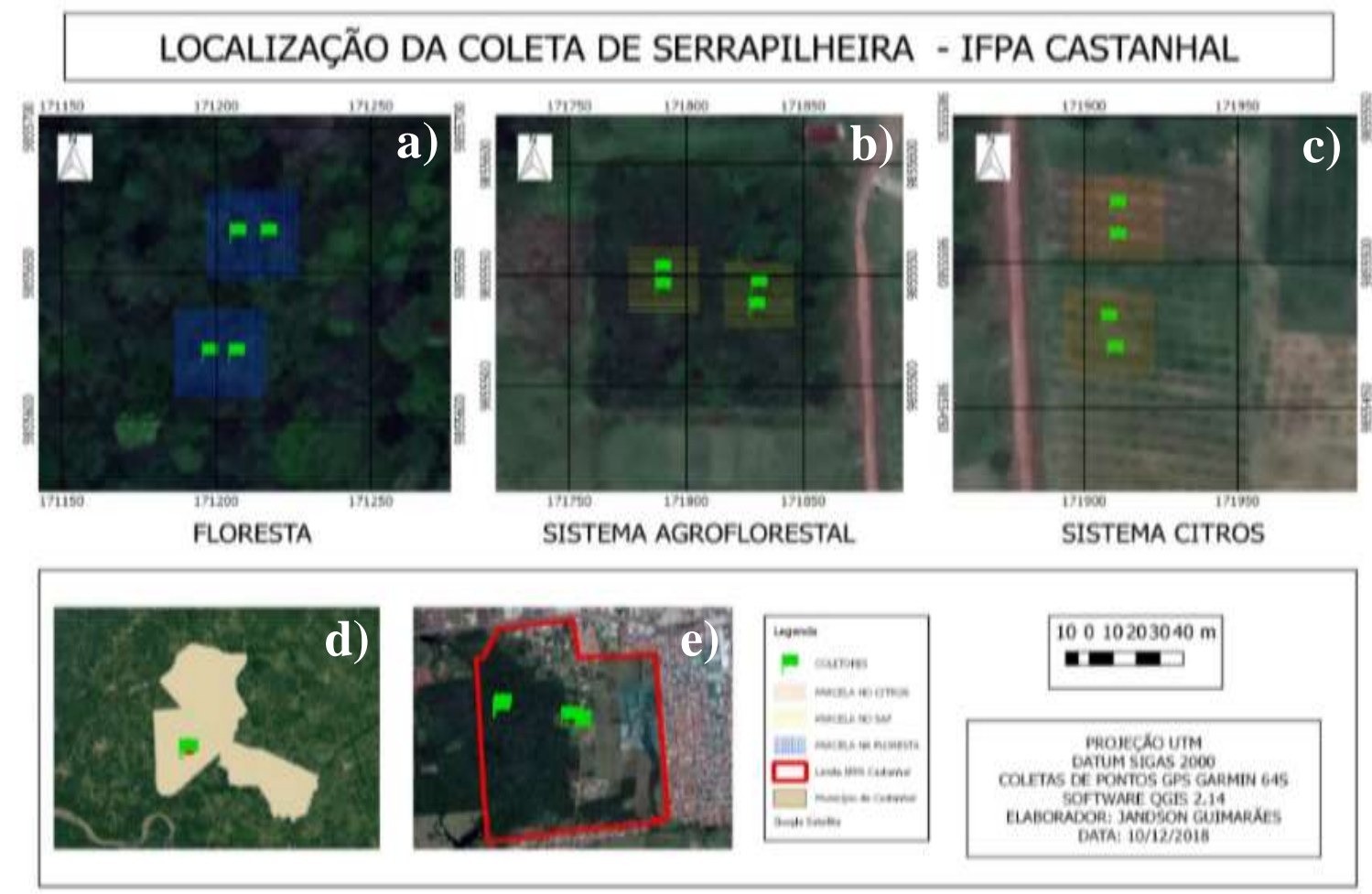

Fonte: Própria

\section{HISTÓRICO DAS ÁREAS}

Área dos citros

A área dos citros foi implantada em janeiro de 2015 após a retirada da cultura de goiaba, a qual possuía como cultivares a Psidium guajava L. (Goiaba paluma) e Psidium guajava L. (Goiaba rica). Foi realizado a poda nos indivíduos como objetivo de favorecer a brotação, no entanto a prática não conseguiu alcançar o objetivo inicial.

A área possui $66 \mathrm{~m}$ de largura e $330 \mathrm{~m}$ de comprimento, sendo composta por Citrus sinensis (L.) Osbeck (Laranja lima), Citrus reticulata Blanco (Tangerina poncã), Citrus reticulata Blanco (Tangerina milharina), e Citrus latifolia, Tanaka (Limão taiti) e acerola ssp. As plantas estão plantadas no espaçamento de $5 \mathrm{mx} 5 \mathrm{~m}$, com adubação no primeiro ano de implantação, e nos seguintes anos não se realizou adubação.

Como prática de manejo ainda foi feita a adubação verde, a partir do consórcio com crotalária. Além disso, usa-se irrigação na área e herbicida para o controle de patógenos, como: pulgões, fungos, minadores, mosca preta e gomose. O arranjo do cultivo e em linhas, totalizando 66 plantas com exceção das tangerinas, as quais possuem 33 plantas para cada uma. 


\section{Área da floresta}

A vegetação da floresta é caracterizada como vegetação densa e está em estágio avançado de sucessão. Em parte da floresta foi realizada a exploração da madeira na década de 70. Em 2010, outra parte da vegetação pegou fogo. No entanto, ainda existem alguns fragmentos com espécies remanescentes de grande porte. Atualmente, não há nenhuma atividade de manejo na área.

\section{Área do SAF cupuaçu}

A área onde foi instalado o sistema agroflorestal era um plantio consorciado de Theobroma grandiflorum Chum. (Cupuaçu) e Musa sp. (Banana), medindo um hectare. A área foi dividida em quatro módulos, cada módulo mediu $2500 \mathrm{~m}^{2}(50 \mathrm{~m}$ x $50 \mathrm{~m})$. As espécies utilizadas para a formação do sistema agroflorestal foram Inga edulis Mart. (Ingá), Sclerolobium paniculatum Vogel (Palheteira), Euterpe oleraceae Mart. (Açaî), Swietenia macrophylla King (Mogno), Tabebuia sp. (Ipê), Carapa guianensis Aubl. (Andiroba), Voacapoua americana Aubl. (Acapu) e Leucaena leucocephala (Lam.) R. de Wit. (Leucena)

As espécies foram plantadas nos espaçamentos de $4 \mathrm{~m}$ x $4 \mathrm{~m}$, entre as linhas do cupuaçu e da banana. Utilizou-se ainda, em algumas parcelas, o Canavalia ensiformis (L.) DC. (Feijão de porco), no espaçamento ( $30 \mathrm{~m}$ x $30 \mathrm{~m}$ ) para ajudar na fertilidade da área. Atualmente, o SAF cupuaçu é usado para fins didáticos.

\section{Delineamento Experimental}

As áreas foram divididas em parcelas de 30 m x 30 m e, em seguida, demarcadas com piquetes nos pontos $10 \mathrm{~m}$ e $20 \mathrm{~m}$, respectivamente. Colocou-se 2 coletores em cada parcela totalizando 4 coletores por sistema, os quais foram padronizados para o lado direito em relação aos piquetes de demarcação (Figura 1).

Figura 1. Demarcação dos coletores de liteira nos sistemas de monocultivo, floresta e SAF.

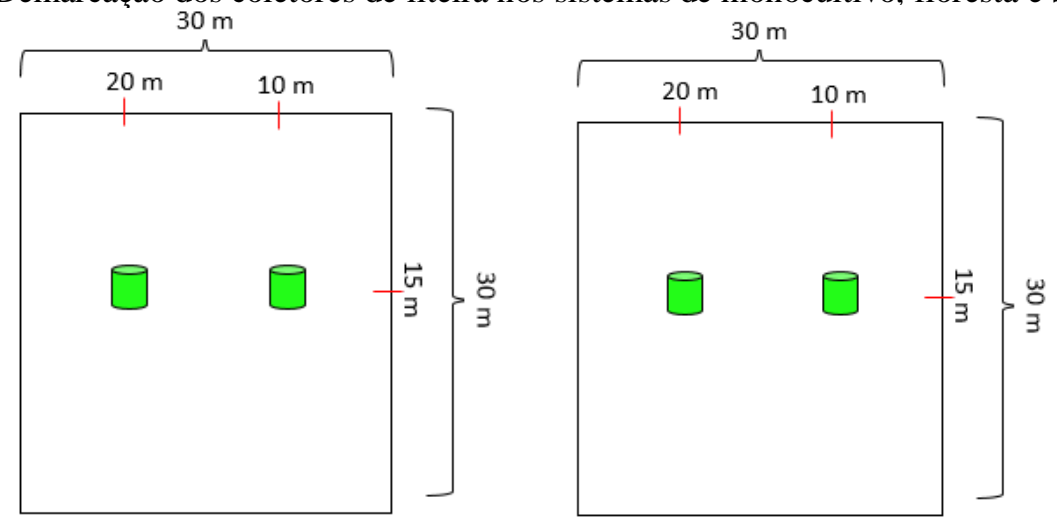

Fonte: Própria 
Para estimar a quantidade de deposição de liteira foram realizadas 3 coletas (repetições) a cada 20 dias ao longo de 60 dias. Essas coletas foram feitas com a utilização de coletores de madeira com dimensões de $1,0 \mathrm{~m}$ x 1,0 m, com fundo em tela de náilon colocados a $0,50 \mathrm{~m}$ do solo. A coleta da liteira acumulada (caracterizada como aquela que fica depositada sobre o solo) foi coletada em uma área de $0,50 \mathrm{~m}$ x $0,50 \mathrm{~m}$. Todo o material foi acondicionado em sacos de papéis para o transporte e secagem.

\section{Análise em laboratório}

As amostras foram secas em estufa $\left(65^{\circ} \mathrm{C}\right)$ por 48 horas, triado em fração não lenhosa (folhas, flores, frutos e sementes) e material lenhoso (galhos e cascas).

Posteriormente, as amostras foram pesadas em balança de precisão, obtendo-se assim o peso da matéria seca em grama por $\mathrm{m}^{2}$. Todas as massas foram anotadas de acordo com a coleta, material lenhoso e não lenhoso, separadamente.

\section{Análise estatística dos dados}

Os dados obtidos em cada amostra da liteira foram analisados por meio do teste não paramétrico de Kruskal-Wallis $(\mathrm{p}<0,05)$ realizado no programa SYSTAT 12.

\section{Resultados e Discussões}

De acordo com as análises houve diferença significativa na produção de material lenhoso entre os três sistemas de manejo $(\mathrm{k}=8,8 ; \mathrm{p}=0,01)$. O SAF apresentou maior produção de material lenhoso $(87,66 \mathrm{~g})$, seguido pela floresta com $25,80 \mathrm{~g}$ e citros produzindo $0,38 \mathrm{~g}$. (Gráfico 1).

Gráfico 1. Medianas da produção de material lenhoso nos sistemas de Citros, Floresta e SAF, no IFPA-

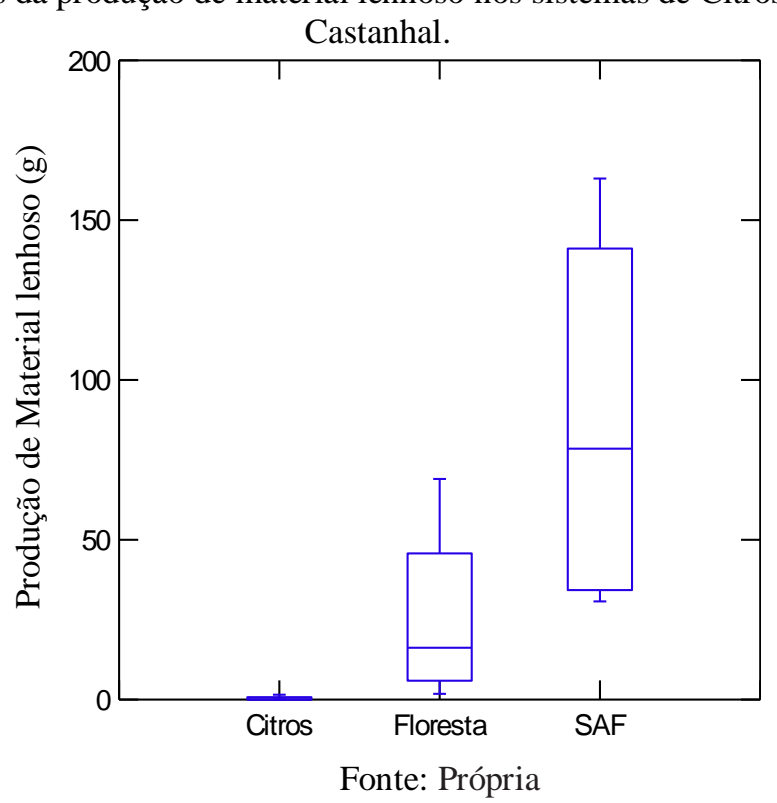


Em relação à produção de material lenhoso acumulado, houve diferença significativa entre os três sistemas de manejo $(\mathrm{k}=8,1 ; \mathrm{p}=0,01)$. O SAF apresentou maior produção de material lenhoso acumulado $(78,75 \mathrm{~g})$, floresta com média de $65,00 \mathrm{~g}$ e citros sem produção de material lenhoso acumulado. (Gráfico 2).

Gráfico 2. Medianas de produção de material lenhoso acumulado nos sistemas de Citros, Floresta e

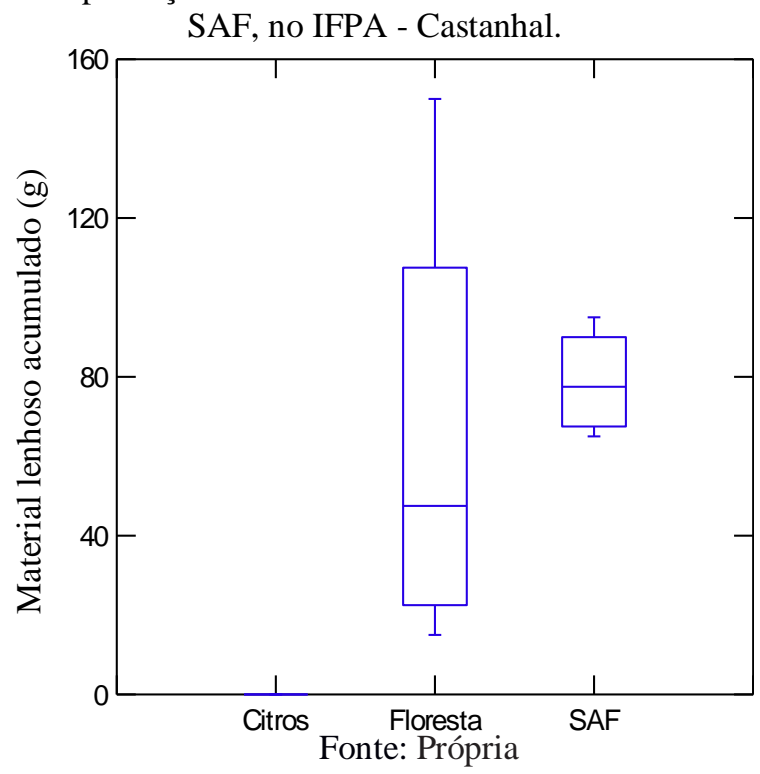

Em relação à decomposição do material lenhoso, houve diferença significativa no coeficiente de decomposição entre os três sistemas de manejo $(k=8,2 ; p=0,01)$. O SAF apresentou maior produção de material lenhoso acumulado, com um coeficiente de decomposição igual à 1,20, seguido pela floresta e citros com valores iguais à respectivamente 0,54 e 0. (Gráfico 3).

Gráfico 3. Medianas do coeficiente de decomposição do material lenhoso nos sistemas de Citros, Floresta e SAF, no IFPA-Castanhal.

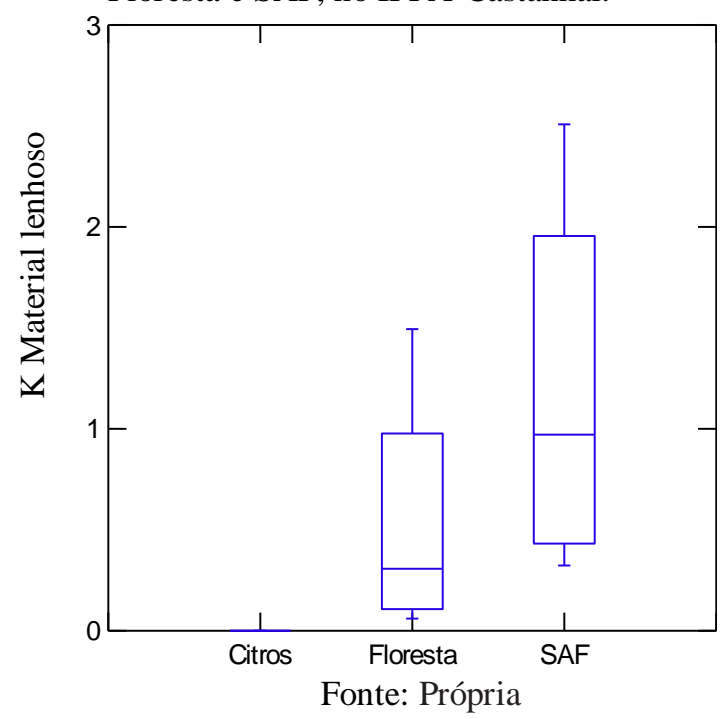


O SAF e a Floresta foram as que apresentaram maiores medias na produção de material não lenhoso com 226,68g e 181,93g respectivamente. Houve diferença significativa na produção de material não lenhoso entre os três sistemas de manejo $(k=7,5 ; p=0,02)$. (Gráfico $4)$.

Gráfico 4. Medianas da produção de material não lenhoso entre os três sistemas de manejo: Citros, Floresta e SAF.

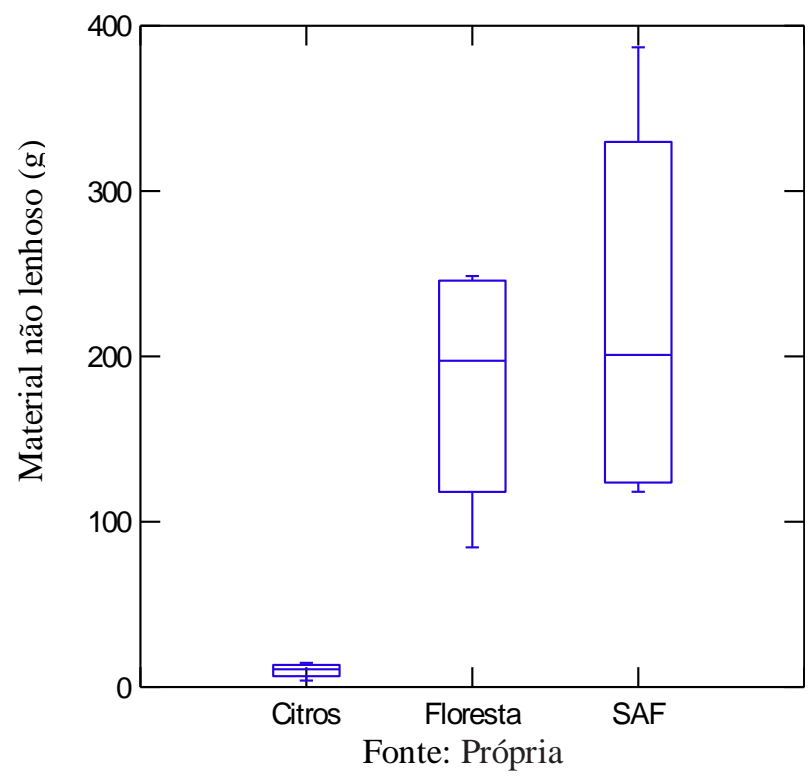

Diferenciou-se significativamente a produção de material não lenhoso acumulado entre os três sistemas de manejo $(\mathrm{k}=8,0 ; \mathrm{p}=0,01)$. A Floresta apresentou maior produção de material não lenhoso acumulado (306,30g), seguido pelo SAF com 195,00g e citros com 66,30g. (Gráfico 5).

Gráfica 5. Medianas da produção de material não lenhoso acumulado nos sistemas de Citros, Floresta e SAF, no IFPA-Castanhal.

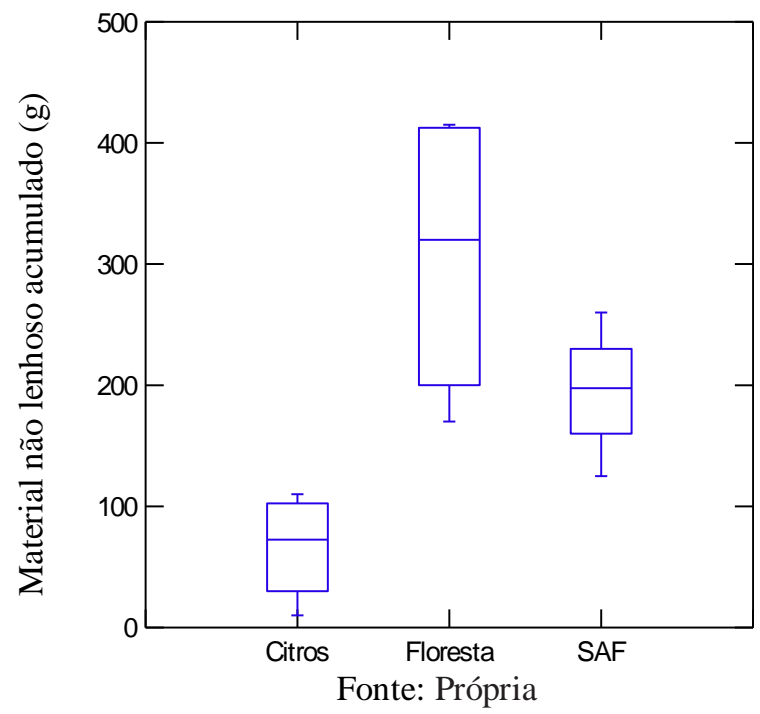


Não houve diferença significativa no coeficiente de decomposição do material não lenhoso entre os três sistemas de manejo $(\mathrm{k}=4,5 ; \mathrm{p}=0,1)$. Apesar de o SAF ter apresentado uma maior média com o $\mathrm{k}=1,1$, o coeficiente de decomposição prevaleceu equivalente nos três sistemas com a floresta apresentando 0,6 e citros com 0,4. (Gráfico 6).

Gráfico 6. Medianas do coeficiente de decomposição do material não lenhoso nos sistemas de Citros, Floresta e SAF, no IFPA-Castanhal.

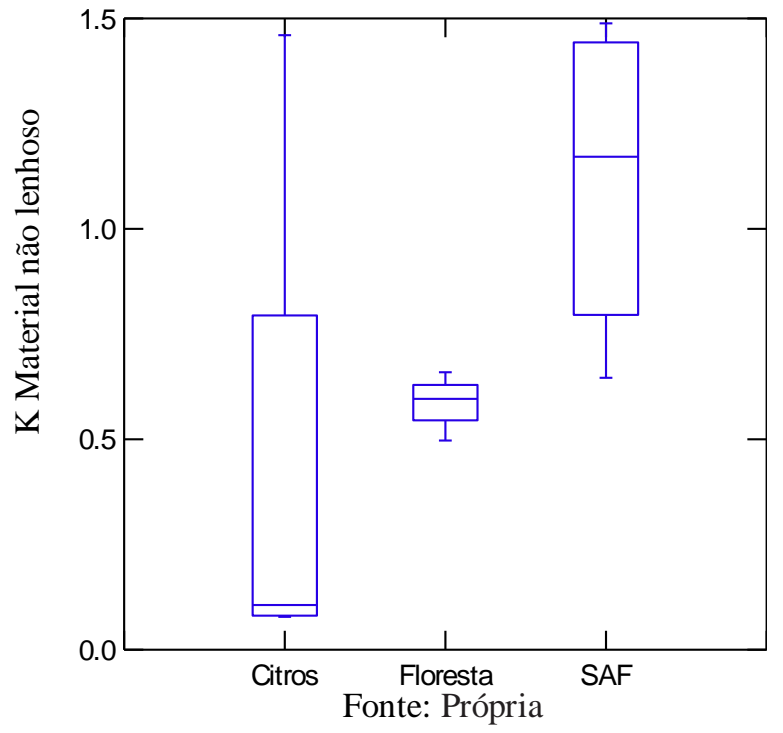

A produção de liteira total foi significativamente diferente entre as três áreas de estudo, assim como verificado no trabalho de Souza (2015) o qual constatou a quantidade de liteira no SAF superior a encontrada na floresta. Tais resultados não coincidiram com os de Esping et al. (2009) e Nascimento et al. (2013), onde apresentaram maior produção de liteira na Floresta do que no SAF. No entanto, o coeficiente de decomposição apresentou-se equivalente aos três sistemas de manejo.

Diversos são os fatores que podem influenciar a produção de liteira: clima, fertilidade do solo, composição de espécies na comunidade, estrutura da vegetação, estádio sucessional da floresta, perturbações antropogênicas na floresta e no entorno (VITOUSEK; SANFORD, 1986; SONGWE et al., 1988; SCHLITTLER et al., 1993; DELITTI, 1995). O trabalho sucedeu-se no inverno e em transição para o verão amazônico, esse fator pode também influenciar consideravelmente na produção de liteira. A maior produção pode estar relacionada com períodos secos (GOLLEY et al., 1978; NUNES, 1980) ou com períodos úmidos (CUNHA et al., 1993; DIAS; OLIVEIRA FILHO, 1997; HINKEL; PANITZ, 1999). 
Considerando os resultados obtidos, verificou-se que os citros apresentaram uma menor produção de material lenhoso, não lenhoso, e o coeficiente de decomposição do material lenhoso acumulado.

A Floresta apresentou no aspecto não lenhoso acumulado uma maior mediana em relação aos outros sistemas. Também, constatou-se na produção de material não lenhoso uma mediana equivalente ao do SAF

Os citros, por exemplo, apresentam-se estruturados em monocultivo. Dada esssa condição, tem-se a diminuição da diversidade vegetal e a consequente redução na diversidade da fauna no sistema, além de apresentar uma incidência direta da chuva e ventos no solo, causando lixiviação dos nutrientes, os quais já se encontram em pequenas quantidades por falta de adubação. Segundo Campanha (2009), a produção de liteira em agroecossistema de monocultivo é menor que em sistema florestal e SAF.

O SAF apresentou uma maior produção de material lenhoso, lenhoso acumulado e um coeficiente de decomposição maior, em relação aos outros dois sistemas. Este fato pode ser explicado pois esse sistema foi manejado no decorrer das nossas coletas, além de que, o SAF apresentava uma estrutura de vegetação com dossel mais fechado comparando-o como os outros.

Outro aspecto que deve-se considerar é que, algumas espécies de Ingá (Inga edulis Mart.) encontravam-se em fase de autodispersão dos frutos, influenciando consideravelmente na pesagem do material do SAF.

Peneireiro (1999) conclui que o manejo da poda é responsável por oferecer estágio sucessional mais avançado, maior disponibilidade de matéria orgânica e maior concentração de nutrientes ao sistema, quando comparado a um agroecossistema que não tem condução de poda, sendo este um manejo essencial para o SAF.

A Floresta foi tida como a testemunha do trabalho, uma vez que, prevalece, atualmente, sem manejo ou outras intervenções humanas. Ela apresentou maior produção de material não lenhoso acumulado. Este fato pode ser explicado, possivelmente, pela razão da Floresta apresentar folhas com maiores dimensões as quais influenciam na pesagem. Também, apresentou o coeficiente de decomposição menor em relação ao SAF, isso traz a reflexão de que a liteira acumulada demora mais para decompor-se nesse sistema, mantendo o material no piso florestal por um tempo maior. Vários fatores podem estar relacionados com a 
decomposição da liteira nos sistemas, destacando-se o clima, a qualidade da serapilheira e a natureza, além da abundância de organismos decompositores (SMITH; BRADFORD, 2003).

\section{Conclusões}

Foram verificadas diferenças significativas no aporte de liteira entre as áreas, com exceção do coeficiente de decomposição do material não lenhoso. Esses fatores: clima, vegetação, composição de espécies na comunidade, fertilidade do solo, fenologia e perturbação antropogênica, tem intrínseca relação com o processo de produção de liteira e decomposição do material no piso dos sistemas. No entanto, são necessários mais estudos, em diferentes épocas do ano e sem intervenções humanas, isto traria mais rigor aos resultados.

\section{Referências}

ABER, J. D.; MELILO, J. M. Terrestrial ecossystems. Orlando: FL.: Reinhart e Wintson. 1978. $428 \mathrm{p}$.

ADAMS, M. A.; ATTIWILL, P. M. Nutrient cycling an nitrogen mineralization in eucalypt forests south-easthern Australia. I. Nutrient Cycling and nitrogen turnover. Plant and Soil, v. 92, n. 3, p. 319-339, 1986.

ANDERSON, J. M.; SWIFT, M. J. Decomposition in tropical forests. Special publications series of the British Ecological Society, p. 503-527, 1983.

BRAY, J.R.; GORHAM, E. Litter production in forests of the word. Advances in Ecological Research. v. 2, n, 1, p. 101-157, 1964.

CÉSAR, O. Produção de serapilheira na mata mesófila semidecídua da Fazenda Barreiro Rico, município de Anhembi, SP. Revista Brasileira de Biologia, v. 53, n. 4, p. 671-681, 1993.

CLIMATE-DATE.ORG. Clima Castanhal. Disponível em: https://pt.climatedata.org/america-do-sul/brasil/para/castanhal-26632/

COSTA, C. C. A., CAMACHO, R. G. V., MACEDO, I. D. \& SILVA, P. C. M. Análise comparativa da produção de serapilheira em fragmentos arbóreos e arbustivos em área de caatinga na Flona de Açu - RN. Revista Árvore, v. 34, n. 2, p. 259-265, 2010.

COSTA, G. S., GAMA-RODRIGUES, A. C. \& CUNHA, G. M. Decomposição e liberação de nutrientes da serapilheira foliar em povoamentos de Eucalyptus grandis no Norte Fluminense. Revista Árvore, v. 29, n. 4, p. 563-570, 2005.

CUEVAS, E.; MEDINA, E. Nutrient dynamics within Amazonian forests. I. Nutrient flux in fine litter fall and efficiency of nutriente utilization. Oecologia, Berlim, v. 68, n. 3, p. 466-472, 
1986.

DELITTI, W. B. C. Estudos de ciclagem de nutrientes: instrumentos para a análise funcional de ecossistemas terrestres. Oecologia Brasiliensis, v. 1, n. 2, p. 469-486, 1995.

ESPIG, S. A.; FREIRE, F. J.; MARANGON, L. C.; FERREIRA, R. L. C.; FREIRE, M. B. G. S.; ESPIG, D. B. Sazonalidade, composição e aporte de nutrientes da serapilheira em fragmento de Mata Atlântica. Revista Árvore, Viçosa, v. 33, n. 5, p. 949 - 956, 2009.

EWEL, J. J. Litter fall and leaf decomposition in a tropical forest succession in eastern Guatemala. Journal of Ecology, v. 64, n. 1, p. 293-308, 1976.

FERREIRA, C. P.; KATO, O. R.; COSTA, C. A. Matéria orgância em sistemas agrícolas na microrregião de Castanhal-Pará. In: Embrapa Amazônia Oriental - Anais (ALICE). In: JORNADA NACIONAL DA PRODUÇÃO CIENTÍFICA EM EDUCAÇÃO PROFISSIONAL E TECNOLÓGICA, 2, 2008, São Luiz. Educação profissional e tecnológica e os desafios do desenvolvimento nacional: livro de resumos. 2. ed. Brasília, DF: Ministério da Educação, Secretaria de Educação Profissional e Tecnológica, 2008.

FIRME PENNA, RODRIGO; DE OLIVEIRA, Rogério Ribeiro. Indicadores de funcionalidade ecossistêmica: integrando os processos de produção e decomposição da serapilheira. Pesquisa, Botânica, São Leopoldo, v. 1, n. 70, p. 213-223, 2017.

GAMA-RODRIGUES, A.C.; MIRANDA, R.C.C. O papel da chuva no fornecimento e reciclagem de nutrientes em um agrossistema de cacau do sul da Bahia, Brasil. Turrialba (IICA), v. 41, n. 4, p. 598-606, 1991.

GOLLEY, F.B. Ciclagem de minerais em um ecossistema de floresta tropical úmida. São Paulo, EPU: EDUSP, 1978. 256 p.

GOIABA Paluma. Toda Fruta, 29 de set. de 2016. Disponível em: https://www.todafruta.com.br/goiaba-paluma. Acesso em: 22 nov. 2018.

GOIABA Rica. Safari Garden, 25 de jun. 2016. Disponível em: https://www.safarigarden.com.br. Acesso em: 22 nov. 2018.

HAAG, H. P. A nutrição mineral e o ecossistema. In: CASTRO, P. R. C., FERREIRA, S. O., YAMADA, T. (Ed.) Ecofisiologia da produção agrícola. Piracicaba: Associação Brasileira para Pesquisa da Potassa e do Fosfato. 1987, 249 p.

JORDAN, C.F.; HERRERA, R. Tropical rain forests: are nutrients really critical? The American Naturalisty, v. 117, n. 2, p. 167-180, 1981.

JORDAN, C.F.; STARK, N. Retención de nutrientes en la estera de raices de un bosque pluvial amazônico. Acta Cient Venezolana, v. 29, n.4, p. 263-267, 1978.

LANDSBERG, J. J.; GOWER, S. T. Applications of physiological ecology to forest management. San Diego: Academic Press. 1997, 354 p. 
LAVELLE, P. Biological processes and productivity of soils in the humid tropics. In: Dickinson, R. E. (ed.). The Geophysiology of Amazônia. Vegetation and Climate Interactions. John Wiley \& Sons. New York. p. 175- 223, 1987.

LEITÃO FILHO, H.F.; PAGANO, S.N.; CÉSAR, O.; TIMONI, J.L.; RUEDA, J.J. Aspectos da ciclagem de nutrientes. In: H.F. Leitão Filho (org.). Ecologia da mata Atlântica em Cubatão. São Paulo, Editora da Universidade Estadual Paulista/ Editora da Universidade de Campinas, p. 129-163, 1993.

LONDSDALE, W.M. Predicting the amout of litterfall in forests of the world. Annals of Botany, v. 61, n. 3, p. 319-324, 1988.

LUIZÃO, F. J.; SCHUBART, H. O. R. Produção e decomposição de liteira em florestas de terra firme da Amazônia Central. Acta Limn. Brasil, v. 1, n. 1, p. 574- 600, 1986.

MARINO, M.C.; FURTADO, J.S.; De Vuono, Y.S. Glossário de Termos Usuais em Ecologia. 1. ed. Academia de Ciências do Estado de São Paulo. v. 24, n.1, p. 97-138, 1980.

MEENTEMEYER, V.; BOX, E.O.; THOMPSON, R. World patterns and amounts of terrestrial plant litter production. BioScience, v. 32, n. 2, p. 125-128, 1982.

MERGURO, M.; VINUEZA, G.N. \& DELITTI, W.B.C. - "Ciclagem de nutrientes minerais na mata mesófila secundária. I Produção e conteúdo de nutrientes minerais do folhedo". Boletim Botânica, universidade São Paulo, v. 7, n. 11, p. 13-31, 1979.

MONTAGNINI, F.; JORDAN, C. F. Reciclaje de nutrientes. In: GUARIGUATA, M. R.; KATTAN, G. H. (Eds.). Ecología y conservación de bosques neotropicais. Cartago: Ediciones LUR, p. 167-191, 2002.

MORAES, R.M.; DELITTI, W.B.C.; STRUFFALDI-DE-VUONO, Y. Litterfall and litter nutrient content two Brazilian Tropical Forests. Revista Brasileira de Botânica, v. 22, n.1, p. 9-16, 1999.

MORELLATO, L. P. C. Nutrient cycling in two south-east Brazilian forests. I- Litterfall and litter standing crop. Journal of Tropical Ecology, v. 8, n. 2, p. 205-215, 1992.

MYERS, R.J.K.; PALM, C.A.; CUEVAS, E.; GUNATILEKE, I.U.N.; BROSSARD, M. The syncronisation of nutrient mineralisation and plant nutrient demand. In: WOOMER, P.L.; SWIFT, P.L. The biological management of tropical soil fertility. John Wiley and John Wiley with the Tropical Soil Biology and Fertility Programme and Sayce Publishing. p. 81-116, 243 p. 1994.

NASCIMENTO, A. F. J.; SILVA, T. O.; SAMPAIO, E. V. S. B.; ARAÚJO FILHO, R. N.; DANTAS, T. V. P. Quantificação de serapilheira em diferentes áreas sob fragmentos do Parque Nacional Serra de Itabaiana, Sergipe. Semina: Ciências Agrárias, Londrina, v. 34, n. 6, p. $3271-3284,2013$. 
OLSON, J.S. Energy storage and the balance of producers and decomposers in ecological systems. Ecology, v. 44, n. 2, p. 223-331, 1963.

PEGADO, C. M. A.; BARBOSA, L. J. N.; MENDES, J. E. M. F.; SOUTO, P. C. \& SOUTO, J. S. Decomposição superficial e subsuperficial de folhas de fava (Phaseolus lunatus L.) na região do Brejo da Paraiba, Brasil. Revista Caatinga, v. 21, n.1, p. 218-223, 2008.

PEIXOTO NETO, Raul Mário da Silva. Processo de decomposição da serrapilheira atuando como mecanismo de facilitação. 2017. 49f. Dissertação (Mestrado em Ecologia) - Centro de Biociências, Universidade Federal do Rio Grande do Norte, Natal, 2017.

PENEIREIRO, F. M. Sistemas agroflorestais dirigidos pela sucessão natural: um estudo de caso. 149f. Dissertação (Mestrado. Ciências Florestais) - Escola Superior de Agricultura "Luiz de Queiroz", Piracicaba, 1999.

PIRES, L. A. et al., Produção, acúmulo e decomposição da serapilheira em uma restinga da Ilha do Mel, Paranaguá, PR, Brasil. Acta Botanica Brasilica, v. 20, n. 1, p. 173-184. 2006.

RICKLEFS, R. A Economia da Natureza: um livro-texto em Ecologia Básica. Guanabara Koogan. 2003. 503 p.

SCHLITTLER, F. H.M.; DE MARINIS, G.; CÉSAR, O. Produção de serapilheira na Floresta do Morro do Diabo, Pontal do Paranapanema - SP. Naturalia, v. 18, n. 1, p. 135-147, 1993.

SMITH, V. C.; BRADFORD, M. A. Litter quality impacts on grassland litter decomposition are differently dependent on soil fauna across time. Applied Soil Ecology, v. 24, n. 2, p. 197203, 2003.

SOUZA, M. C. S. et al. Funcionalidade Ecológica De Sistemas Agroflorestais Biodiversos: Uso Da Serapilheira Como Indicador Da Recuperação De Áreas De Preservação Permanente. Floresta, Curitiba, PR, v. 46, n. 1, p. 75 - 82, 2015.

SPAIN, A.V. Litterfall and the standing crop of litter in three tropical Australian rainforests. Journal of Ecology, v. 72, n. 3, p. 947-961, 1984.

SUNDARAPADIAN, S.M.; SWAMY, P.S. Litter production and leaf-litter decomposition of selected tree species in tropical forests at Kodayar in the Western Ghats, India. Forest Ecology and Management. v. 123, n. 2, p. 231-244, 1999.

TEIXEIRA, L. B.; BASTOS, J. B. Matéria orgânica nos ecossistemas de floresta priméiria e pastagens na Amazônia Central. Boletim de Pesquisa, 99. (EMBRAPA-CPATU), Belém. 26 p. 1989.

TEIXEIRA, M. B.; LOSS, A.; PEREIRA, M. G. \& PIMENTEL, C. Decomposição e ciclagem de nutrientes dos resíduos de quatro plantas de cobertura do solo. Idesia (Arica), v. 30, n. 1, p. 55-64, 2012.

TIAN, G., KANG, B. T.; BRUSSAARD, L. Biological effects of plant residues with contrasting 
chemical compositions under humid tropical conditions- decomposition and nutrient release. Soil Biology and Biochemistry, v. 24, n. 10, p. 1051-1060, 1992.

VIDAL, MARIANA MORAIS et al. Produção de serapilheira em floresta Atlântica secundária numa paisagem fragmentada (Ibiúna, SP): importância da borda e tamanho dos fragmentos. Revista brasileira de Botânica, v. 30, n. 3, p. 521-532, 2007.

VITOUSEK, P.M. Litterfall, nutrient cycling and nutrient limitation in tropical forests. Ecology, v. 65, n.1, p. 285-298, 1984.

VITOUSEK, P.M.; SANFORD, R.L.Jr. Nutrient cycling in moist tropical forest. Annual Review of Ecology and Systematics, v. 17, n, 1, p. 137-167, 1986. 